\title{
Quantification of microdosed oral yohimbine and its major metabolite in human plasma in the picogram range
}

\author{
Manuela Vay ${ }^{1}$ iD, Max Sauter ${ }^{1}$, Gerd Mikus $^{1}$ \& Jürgen Burhenne*,1 \\ ${ }^{1}$ Department of Clinical Pharmacology \& Pharmacoepidemiology, Im Neuenheimer Feld 410, Heidelberg University Hospital, \\ Heidelberg 69120, Germany \\ *Author for correspondence: Tel.: +49 622156 36395; Fax: +49 622156 5832; juergen.burhenne@med.uni-heidelberg.de
}

\begin{abstract}
Aim: Pharmacokinetics after oral microdosing of the anticipated CYP2D6 substrate yohimbine and its metabolite 11-OH-yohimbine is potentially useful for drug-drug interaction trials and profiling of CYP2D6 enzyme activity. Materials $\&$ methods: We developed an ultrasensitive ultra performance liquid chromatography coupled to tandem mass spectrometry assay for quantification of yohimbine and its main metabolite $11-\mathrm{OH}$-yohimbine in plasma with a linear calibration range of $5-2500 \mathrm{pg} / \mathrm{ml}$ and validated it according to US FDA's and EMA's guidelines. Sample preparation was performed using fast liquidliquid extraction. The assay was applied for the determination of concentrations of yohimbine and 11$\mathrm{OH}$-yohimbine in plasma after oral administration of $50 \mu \mathrm{g}$ yohimbine to two subjects. Conclusion: Ultrasensitive quantification of yohimbine and its metabolite enables the determination of their concentrations in plasma after microdosing which would be applicable to use in CYP2D6 phenotyping.
\end{abstract}

First draft submitted: 28 May 2019; Accepted for publication: 25 July 2019; Published online: 14 August 2019

Keywords: CYP2D6 phenotyping $\bullet$ microdosing $\bullet$ tandem mass spectrometry $\bullet$ ultra performance liquid chromatography $\bullet$ yohimbine

The pharmacologically sparsely described $\alpha_{2}$-receptor antagonist yohimbine is licensed in Germany for the treatment of erectile dysfunction with recommended daily doses of $10-30 \mathrm{mg}$. Its mechanism of action is not yet fully understood [1], yet impacts on the penile venous blood vessels [2] or in the central nervous system [3] are considered to play a part. While yohimbine is losing importance for erectile dysfunction therapy, it is found in many dietary supplements for enhancing libido, for weight loss and as aid for bodybuilding [4].

Yohimbine is metabolized primary into the active metabolite 11-OH-yohimbine and to a smaller extent into 10-OH-yohimbine [5-7]. In vitro data indicate that CYP2D6 is likely to be involved in the metabolism of yohimbine to 11-OH-yohimbine [8]. CYP2D6 undergoes a genetic polymorphism resulting in highly variable activity with approximately $10 \%$ of the European population being CYP2D6 poor metabolizers [9]. Monitoring enzyme activity during early phase clinical trials and also in drug-drug interaction trials is gaining importance since the introduction of microdosing. A microdose is characterized as being $1 / 100$ th of the pharmacologically active dose or $100 \mu \mathrm{g}$, whichever is the lower amount and causes no demonstrable pharmacodynamic effect. Such a dosing regimen improves the safety of participants in drug-drug interaction trials and is advantageous because the microdosed probe drugs do not act as perpetrators and thus can be used in substrate cocktails.

Only a few analytical assays to quantify yohimbine have been described in the literature and these used liquidliquid extraction with alkaline buffer and organic solvents for sample preparation with large plasma volumes of 0.5-2 ml. Normal phase or reversed-phase C8 columns for liquid chromatography were coupled to electrochemical or fluorimetric detection. A recent method used protein precipitation for extraction and quantified yohimbine via LC-MS/MS showing an lower limit of quantification (LLOQ) of $0.1 \mathrm{ng} / \mathrm{ml}$ for yohimbine. 11-OH-yohimbine has been quantified only by fluorimetric detection with an LLOQ of $1 \mathrm{ng} / \mathrm{ml}[6,10]$.

To prove the suitability of microdosed oral yohimbine administration for potential drug-drug interaction trials, we developed an ultra performance liquid chromatography coupled to tandem mass spectrometry (UPLC- 
MS/MS) assay with positive electrospray ionization and multiple reaction monitoring (MRM) for simultaneous quantification of yohimbine and 11-OH-yohimbine in the picogram range utilizing liquid-liquid extraction for sample preparation and validated the assay according to the pertinent guidelines of US FDA and EMA [11,12]. The method was successfully applied for the quantification of concentrations of yohimbine and 11-OH-yohimbine in plasma from two healthy subjects who received a single oral dose of $50 \mu \mathrm{g}$ yohimbine.

\section{Materials \& methods}

Drugs, chemicals, solvents \& materials

Yohimbine hydrochloride was kindly provided by Herband Pharmachemicals (Gengenbach, Germany). Pierre Fabre laboratoires (Toulouse, France) kindly donated $11-\mathrm{OH}$-yohimbine $(5.1 \mathrm{mg}, 98 \%$ purity) and internal standard (IS; $\left[{ }^{13} \mathrm{C}^{2} \mathrm{H}_{3}\right]$-yohimbine) was purchased from Alsachim (Illkirch, France). Yohimbine tablets for oral microdoses (Yohimbinum hydrochloricum D4 ${ }^{\circledR}$ ) were purchased from Deutsche Homöopathie Union (Karlsruhe, Germany). Boric acid and ammonium acetate were obtained from Sigma-Aldrich (Darmstadt, Germany). Acetonitrile (ACN), isopropanol, acetic acid, formic acid (FA), sodium hydroxide, methyl tert-butyl ether (TBME) and methanol were purchased from Biosolve BV (Valkenswaar, The Netherlands) in the highest available purity (ULC/MS grade). For calibration standards, quality controls (QCs) and validation-purpose drug-free plasma from healthy subjects were obtained at the local blood bank.

\section{Clinical study \& preparation of plasma samples}

The competent authority (Bundesinstitut für Arzneimittel und Medizinprodukte, Germany) and the Ethics Committee of the Medical Faculty of Heidelberg (Germany) approved the clinical trial protocol (EudraCT 2017001801-34). The trial was conducted at the Clinical Research Unit (KliPS, certified according to ISO 9001:2015) of the Department of Clinical Pharmacology and Pharmacoepidemiology in accordance with German legal requirements, good clinical practice guidelines and Declaration of Helsinki. Healthy subjects supplied written consent before participation. After oral administration of $50 \mu \mathrm{g}$ yohimbine, blood samples were taken into heparinized tubes (7.5 ml, S-Monovette ${ }^{\circledR}$, Sarstedt AG, Nümbrecht, Germany) before and at $0.25,0.5,0.75,1,1.25,1.5$, $1.75,2,2.5,3,4,5,6,8,10,12$, as well as $24 \mathrm{~h}$ after administration. After centrifugation $\left(3600 \times g, 10 \mathrm{~min}, 4^{\circ} \mathrm{C}\right)$ plasma samples were stored in aliquots at $-25^{\circ} \mathrm{C}$ until analysis.

\section{Standard preparation}

For the IS solution $\left[{ }^{13} \mathrm{C}^{2} \mathrm{H}_{3}\right]$-yohimbine was dissolved in ACN/water $(1: 1, \mathrm{v} / \mathrm{v}, 1 \mathrm{mg} / \mathrm{ml})$ and diluted to a concentration of $100 \mathrm{ng} / \mathrm{ml}$. For standard stock solutions, yohimbine $(3.11 \mathrm{mg})$ and $11-\mathrm{OH}$-yohimbine $(2.05 \mathrm{mg})$ were dissolved in ACN/water (1:1, v/v), subsequently combined and further diluted to yield calibration working solutions.

Stock solutions for QC samples were weighed independently and prepared as described for calibration solutions.

\section{Calibration \& QC samples}

Samples for calibration and QCs were prepared by spiking blank plasma $(100 \mu \mathrm{l})$ with $25 \mu \mathrm{l}$ of the corresponding calibration or QC working solutions to provide calibration concentrations of 5, 10, 50, 100, 250, 500, 1000 and $2500 \mathrm{pg} / \mathrm{ml}$ and QC concentrations of 8.50, 199 and $1986 \mathrm{pg} / \mathrm{ml}$ (yohimbine) and 9.74, 195 and $1948 \mathrm{pg} / \mathrm{ml}$ (11-OH-yohimbine).

\section{Liquid-liquid extraction for plasma sample preparation}

The respective working solutions $(25 \mu \mathrm{l})$ were spiked to blank plasma $(100 \mu \mathrm{l})$ in plastic tubes $(10 \mathrm{ml})$ for preparation of calibration and QC samples. For volume compensation study samples $(100 \mu \mathrm{l})$ were spiked with ACN/water $(25 \mu \mathrm{l}, 1: 1, \mathrm{v} / \mathrm{v})$. Each sample was additionally spiked with IS solution $(25 \mu \mathrm{l})$. After addition of borate buffer $\mathrm{pH} 9(100 \mu \mathrm{l})$ samples were vortexed and TBME $(2 \mathrm{ml})$ was added. After shaking $(10 \mathrm{~min})$ and centrifugation $(10 \mathrm{~min}, 3600 \times \mathrm{g})$, the organic phase $(1.5 \mathrm{ml})$ was transferred into glass tubes and evaporated to dryness under a stream of nitrogen at $40^{\circ} \mathrm{C}$. UPLC eluent $(100 \mu \mathrm{l}$, ACN/water; 5:95 v/v with $0.1 \% \mathrm{FA})$ was used for reconstitution of the residue. After shaking on a vortex-mixer and sonication, the solution was transferred to a 96-well collection plate (800 $\mu$ l, Waters, MA, USA). 


\section{Instrumental analysis parameters}

The UPLC-MS/MS system (Waters) consisted of an Acquity UPLC ${ }^{\circledR}$ system including a Waters Sample Manager, binary solvent manager, cooled sample trays, integrated column heater and degasser coupled to a triple-stage quadrupole mass spectrometer (Xevo TQ-S, Waters) equipped with Z-spray ionization.

Chromatographic separation was achieved at $40^{\circ} \mathrm{C}$ on a Waters Acquity BEH C18 column $(1.7 \mu \mathrm{m}$, $2.1 \times 50 \mathrm{~mm}$ ) including an integrated filter disc. The injection volume was $20 \mu \mathrm{l}$ and the eluent consisted of water including $5 \% \mathrm{ACN}$ and $0.1 \% \mathrm{FA}(\mathrm{A})$ and $\mathrm{ACN}$ including $0.1 \% \mathrm{FA}(\mathrm{B})$ at a flow rate of $0.5 \mathrm{ml} / \mathrm{min}$. The gradient started at $95 \% \mathrm{~A} / 5 \% \mathrm{~B}$ and held for $0.1 \mathrm{~min}$ and then changed within 2 min to $5 \% \mathrm{~A} / 95 \% \mathrm{~B}$, kept at these conditions until $2.5 \mathrm{~min}$ and changed at $3 \mathrm{~min}$ back to $95 \% \mathrm{~A} / 5 \% \mathrm{~B}$. Ionization parameters were as follows: spray voltage $500 \mathrm{~V}$, cone voltage $25 \mathrm{~V}$, cone gas flow (N2) $150 \mathrm{l} / \mathrm{h}$, desolvation temperature $600^{\circ} \mathrm{C}$, desolvation gas flow $\left(\mathrm{N}_{2}\right) 1000 \mathrm{l} / \mathrm{h}$, source temperature $150^{\circ} \mathrm{C}$. Tuning of the Xevo TQ-S to yohimbine, 11-OH-yohimbine and labeled IS was performed automatically using the integrated software package (MassLynx V4.1) and IntelliStart standard procedures. For collision induced decomposition (CID), argon was used as collision gas at a flow rate of $0.15 \mathrm{ml} / \mathrm{min}$. MS/MS transitions for MRM in positive ionization mode were $\mathrm{m} / z 355.2 \rightarrow 144.2$ at $30 \mathrm{~V}$ for yohimbine (quantifier), $m / z 355.2 \rightarrow 212.2$ at $30 \mathrm{~V}$ for yohimbine (qualifier), $m / z 359.2 \rightarrow 144.2$ at $30 \mathrm{~V}$ for $\left[{ }^{13} \mathrm{C}^{2} \mathrm{H}_{3}\right]$-yohimbine and $m / z 371.2 \rightarrow 160.2$ at $24 \mathrm{~V}$ for $11-\mathrm{OH}$-yohimbine (quantifier) and $m / z 371.2 \rightarrow 212.2$ at $24 \mathrm{~V}$ for $11-\mathrm{OH}$-yohimbine (qualifier). Dwell time for each transition was $0.063 \mathrm{~s}$ resulting in a total cycle time of $0.315 \mathrm{~s}$.

\section{Validation}

Validation of the assay was conducted in accordance with the guidelines recommended by the FDA and EMA. Three validation batches were analyzed, each including eight calibration samples and 24 QC samples (LLOQ; QC A; QC B; QC C). Accuracy (\%) was calculated as ratio of the average concentration values measured in respective batches divided by the nominal concentrations. Precision (\%) was determined as ratio of standard deviation and the mean sample concentration. For determination of selectivity blank plasma of six different individuals was analyzed as described without spiking analyte or IS. Recovery was investigated by comparison of peak area of QC samples divided by the corresponding peak areas of blank plasma spiked with the respective QC solutions after extraction (100\% analyte in the same matrix). Stability was demonstrated by determination of QCs after three freeze-thaw cycles, lasting at least $24 \mathrm{~h}$. Stability of extracts during analyses was proven by repeated measurement of QCs after staying in the autosampler at $15^{\circ} \mathrm{C}$ over night.

\section{Statistical \& calculation methods}

Calibration plasma samples and their respective quantifier MRM transitions were used to generate calibration curves for yohimbine and 11-OH-yohimbine. TargetLynx V4.1 (Waters) software was used to calculate peak area ratios of analytes and IS and weighted linear regression $(1 / \mathrm{x})$ was performed. For pharmacokinetic data analysis Kinetica 5.0 (Thermo Fisher Scientific, MA, USA) was used and graphs created by Prism 7.02 (Graphpad, CA, USA).

\section{Results \& discussion}

\section{Sample preparation}

Best results for sample preparation were achieved by liquid-liquid extraction using borate buffer $\mathrm{pH} 9$ and TBME. Analyte plasma recovery was determined from QCs A-C in triplicates and ranged between 80.7 and $108 \%$ for yohimbine, 86.7 and $98.2 \%$ for $11-\mathrm{OH}$-yohimbine and 92.4 and $110 \%$ for $\left[{ }^{13} \mathrm{C}^{2} \mathrm{H}_{3}\right]$-yohimbine. These consistent recoveries of yohimbine and its metabolite 11-OH-yohimbine confirm the suitability of the IS to account for the extraction characteristics of both analytes which resulted in precise quantification despite no separate isotopically labeled standard for 11-OH-yohimbine.

\section{Chromatography \& mass spectrometry}

A Waters BEH C18 UPLC column $(1.7 \mu \mathrm{m})$ and a fast gradient from 5 to $95 \%$ organic solvent achieved baseline separated peaks and a short run time of 3 min (Figure 1). The fast and steep gradient was necessary to achieve sharp peaks for 11-OH-yohimbine because slower gradients led to a substantial increase in peak width impeding sensitive quantification. 


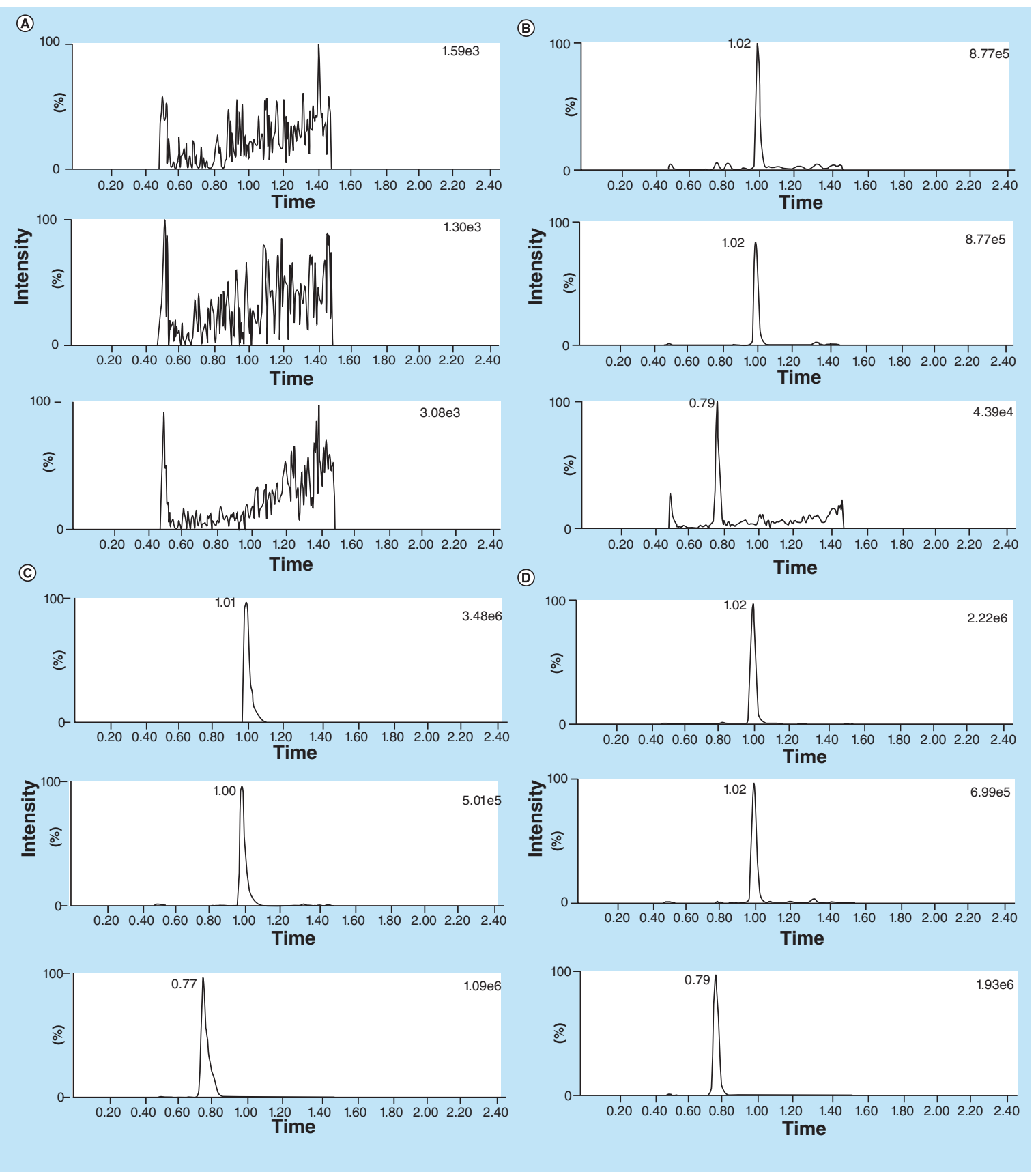

Figure 1. Ultra performance liquid chromatography coupled to tandem mass spectrometry chromatograms of processed plasma samples with mass transitions of yohimbine (top trace), [13C2H3]-yohimbine (middle trace), 11-OH-yohimbine (bottom trace) shown separately. (A) Blank plasma sample. (B) Plasma sample spiked at lower limit of quantification concentration. (C) Plasma sample spiked at quality control B concentration. (D) Plasma sample of subject $1,2 \mathrm{~h}$ after oral administration of $50 \mu \mathrm{g}$ yohimbine (calculated yohimbine concentration: $79.9 \mathrm{pg} / \mathrm{ml}$ and calculated 11-OH-concentration: $218 \mathrm{pg} / \mathrm{ml}$ ).

Positive electrospray ionization generated intense $[\mathrm{M}+\mathrm{H}]^{+}$signals when using acidic eluents for both analytes and the IS. The $[\mathrm{M}+\mathrm{H}]^{+}$signal for yohimbine at $m / z 355.2$ showed highest intensity and hence was used for quantification. For the IS and 11-OH-yohimbine, a mass shift of $m / z 4$ for the IS and $m / z 16$ for the corresponding $[\mathrm{M}+\mathrm{H}]^{+}$ions used for quantification were observed at $m / z 359.2$ and $m / z 371.2$, respectively.

CID of yohimbine generated two abundant product ions at $m / z 144.2$ and $m / z 212.2$ with the $\mathrm{m} / z 144.2$ showing considerable higher intensity (Figure 2). The same pattern was observed for the IS with a mass shift of 


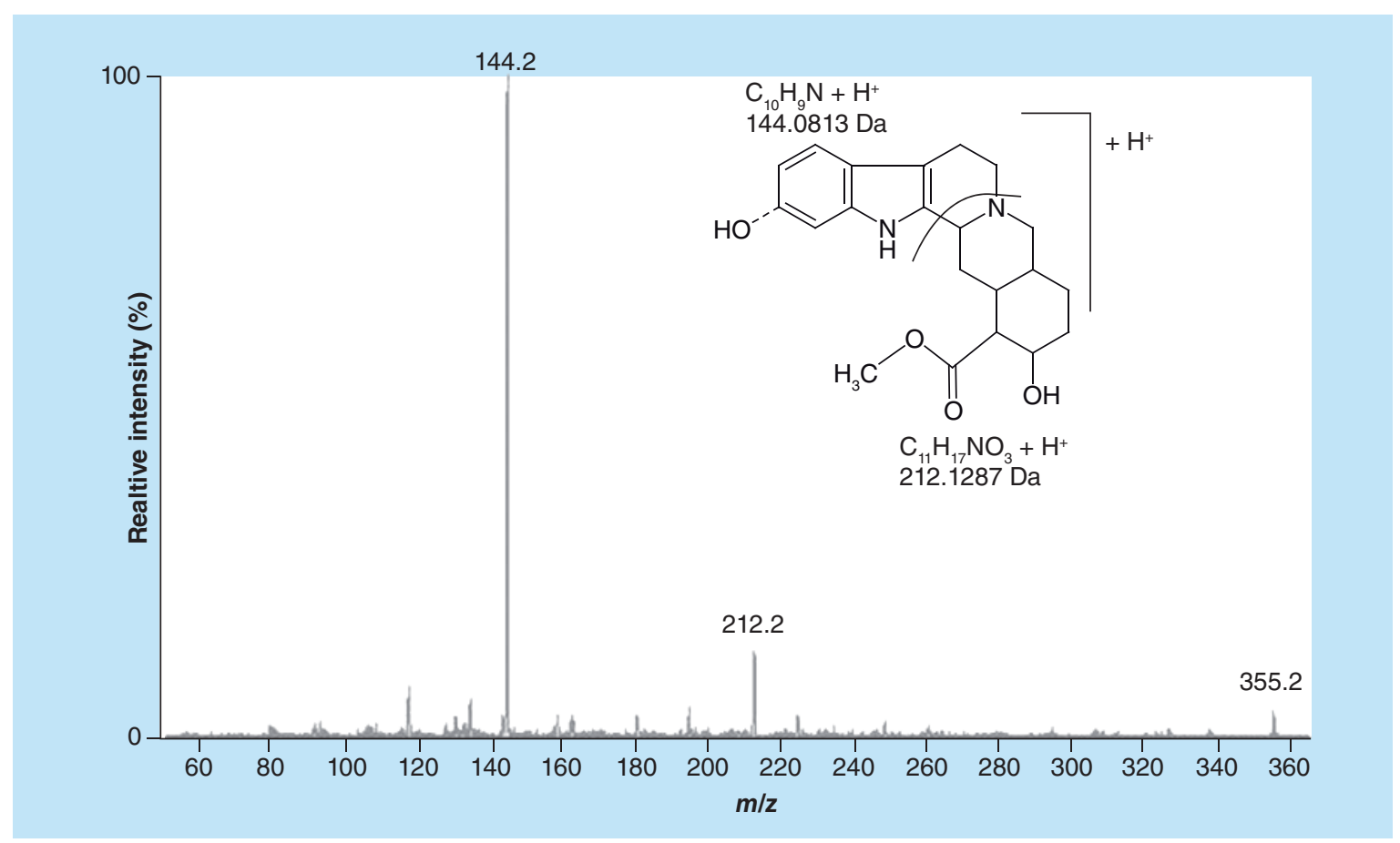

Figure 2. Positive product ion spectrum of the $[\mathrm{M}+\mathrm{H}]^{+}$yohimbine signal at $m / z 355$. In the chemical structure, the star depicts the position of the methyl group labeled with ${ }^{13} \mathrm{C}$ and ${ }^{2} \mathrm{H}_{3}$ in the internal standard and the position of the hydroxyl group in the metabolite 11-OH-yohimbine is implied by the dashed line.

$4 \mathrm{Da}$ at the less intense fragment yielding product ions at $m / z 144.2$ and $m / z$ 216.2. 11-OH-yohimbine also showed two main product ions at $m / z 160.2$ and $m / z 212.2$ in CID. The mass shift of $16 \mathrm{Da}$ for the more intense fragment corresponds to the additional oxygen of the hydroxyl group in comparison to yohimbine. Due to the almost exclusive abundance of the two product ions, only one major decomposition is anticipated for yohimbine as well as 11-OH-yohimbine which is depicted in Figure 2 producing the two observed product ions, most likely as $[\mathrm{M}+\mathrm{H}]^{+}$signals.

The more intense fragment was hence chosen for quantification while the less intense product ion served as a qualifier transition increasing specificity of the detection. Identity of the product ions was investigated by high resolution mass spectrometry using a Xevo-G2-XS quadrupole time of flight mass spectrometer (Waters) and in accordance with the suspected structures: product ions of yohimbine, 11-OH-yohimbine and $\left[{ }^{13} \mathrm{C}^{2} \mathrm{H}_{3}\right]$-yohimbine showed $m / z$ values of 144.0826 and $212.1301,160.0775$ and 212.1300 , as well as 144.0836 and 216.1512; in accordance with the theoretical $\mathrm{m} / z$ values of 144.0813 and $212.1287,160.0757$ and 212.1287 , as well as 144.0808 and 216.1509, respectively.

\section{Validation}

Validation results fully complied with the requirements of FDA's and EMA's guidelines for bioanalytical method validation. Absence of interference in blank plasma of six different individuals proved selectivity of the assay (Figure 1). Within-batch accuracies for analytes at the LLOQ level ranged from 102 to 117\% with maximum deviation of precision of $10.8 \%$. Batch-to-batch accuracies ranged from 99.8 to $111 \%$ with a maximum precision deviation of $8.40 \%$. For QC A-C within-batch accuracies ranged from 86.0 to $106 \%$ with a maximum precision deviation of $4.60 \%$ and batch-to-batch accuracies from 87.2 to $104 \%$ with a maximum deviation of precision of $6.30 \%$ for both analytes. Complete results of the assay validation are shown in Table 1 .

Correlation coefficients for linear regression were $>0.997$ for yohimbine and 11-OH-yohimbine (weighting $1 / \mathrm{x}$ ). Stability in plasma samples was demonstrated for QC A-C after three freeze-and-thaw cycles with corresponding accuracies ranging from 94.8 to $114.9 \%$, and plasma extracts were stable after remaining in the autosampler at $15^{\circ} \mathrm{C}$ for $24 \mathrm{~h}$. 


\begin{tabular}{|c|c|c|c|c|}
\hline Within-batch & LLOQ & QCA & QCB & QCC \\
\hline \multicolumn{5}{|l|}{ Yohimbe } \\
\hline Concentration $(\mathrm{pg} / \mathrm{ml})$ & 5.00 & 8.50 & 199 & 1986 \\
\hline \multicolumn{5}{|l|}{ 1: } \\
\hline - Mean (pg/ml) & 5.42 & 7.52 & 176 & 1727 \\
\hline - Accuracy (\%) & 8.38 & -11.6 & -11.2 & -13.0 \\
\hline - Precision (\%) & 2.40 & 1.90 & 1.50 & 10.8 \\
\hline \multicolumn{5}{|l|}{ 2: } \\
\hline - Mean (pg/ml) & 4.76 & 8.80 & 171 & 1708 \\
\hline - Accuracy (\%) & -4.88 & 3.47 & -14.0 & -14.0 \\
\hline - Precision (\%) & 3.80 & 2.70 & 0.70 & 0.50 \\
\hline \multicolumn{5}{|l|}{ 3: } \\
\hline - Mean (pg/ml) & 4.85 & 8.37 & 183 & 1757 \\
\hline - Accuracy (\%) & -3.01 & -1.49 & -7.82 & -11.6 \\
\hline - Precision (\%) & 7.10 & 2.80 & 1.80 & 1.90 \\
\hline \multicolumn{5}{|l|}{ Batch-to-batch: } \\
\hline - Mean (pg/ml) & 4.99 & 8.26 & 177 & 1731 \\
\hline - Accuracy (\%) & -0.20 & -2.80 & -10.8 & -12.8 \\
\hline - Precision (\%) & 8.40 & 6.30 & 3.50 & 1.80 \\
\hline \multicolumn{5}{|l|}{$>11-\mathrm{OH}-$ yohimbine } \\
\hline Concentration $(\mathrm{ng} / \mathrm{ml})$ & 5.0 & 9.74 & 195 & 1948 \\
\hline \multicolumn{5}{|l|}{ 1: } \\
\hline - Mean (pg/ml) & 5.87 & 10.3 & 204 & 2069 \\
\hline - Accuracy (\%) & 17.2 & 5.59 & 4.77 & 6.20 \\
\hline - Precision (\%) & 1.70 & 4.60 & 4.20 & 2.30 \\
\hline \multicolumn{5}{|l|}{ 2: } \\
\hline - Mean (pg/ml) & 5.75 & 10.1 & 199 & 1987 \\
\hline - Accuracy (\%) & 15.0 & 3.39 & 2.25 & 2.00 \\
\hline - Precision (\%) & 2.50 & 2.10 & 2.40 & 1.10 \\
\hline \multicolumn{5}{|l|}{ 3: } \\
\hline - Mean (pg/ml) & 5.09 & 9.65 & 203 & 1956 \\
\hline - Accuracy (\%) & 1.71 & -0.98 & 3.99 & 0.41 \\
\hline - Precision (\%) & 2.70 & 2.90 & 4.40 & 3.30 \\
\hline \multicolumn{5}{|l|}{ Batch-to-batch: } \\
\hline - Mean (pg/ml) & 5.54 & 9.99 & 202 & 2001 \\
\hline - Accuracy (\%) & 10.7 & 2.50 & 3.60 & 2.70 \\
\hline - Precision (\%) & 6.70 & 3.80 & 3.60 & 3.00 \\
\hline
\end{tabular}

Pharmacokinetics in two healthy subjects after administration of $50 \mu \mathrm{g}$ oral yohimbine

Pharmacokinetics of oral yohimbine microdoses were determined in two healthy subjects (Figure 3) who had been genotyped to show they had markedly different CYP2D6 enzyme activity to evaluate the suitability of the assay for the investigation of the potential of yohimbine for profiling of CYP2D6 enzyme activity. The calibration range from 5 to $2500 \mathrm{pg} / \mathrm{ml}$ was sufficient for the investigation of plasma concentrations after administration of oral microdoses. Yohimbine half-life was substantially different in the two subjects, yet in accordance with the determined enzyme activity by genotyping and the amount of metabolite generated. The pharmacokinetics of yohimbine and its metabolite in both subjects are shown in Table 2.

Subject 1 possessing normal enzyme activity achieved maximum plasma concentrations of yohimbine and 11$\mathrm{OH}$-yohimbine $15 \mathrm{~min}$ after administration while subject 2 (genotyped as a poor metaboliser) did after $45 \mathrm{~min}$. Further, maximum plasma concentration of yohimbine was more than threefold higher in subject 2 while subject 1 showed systematic exposure twice as much metabolite compared with subject 2 which is in line with the expected 


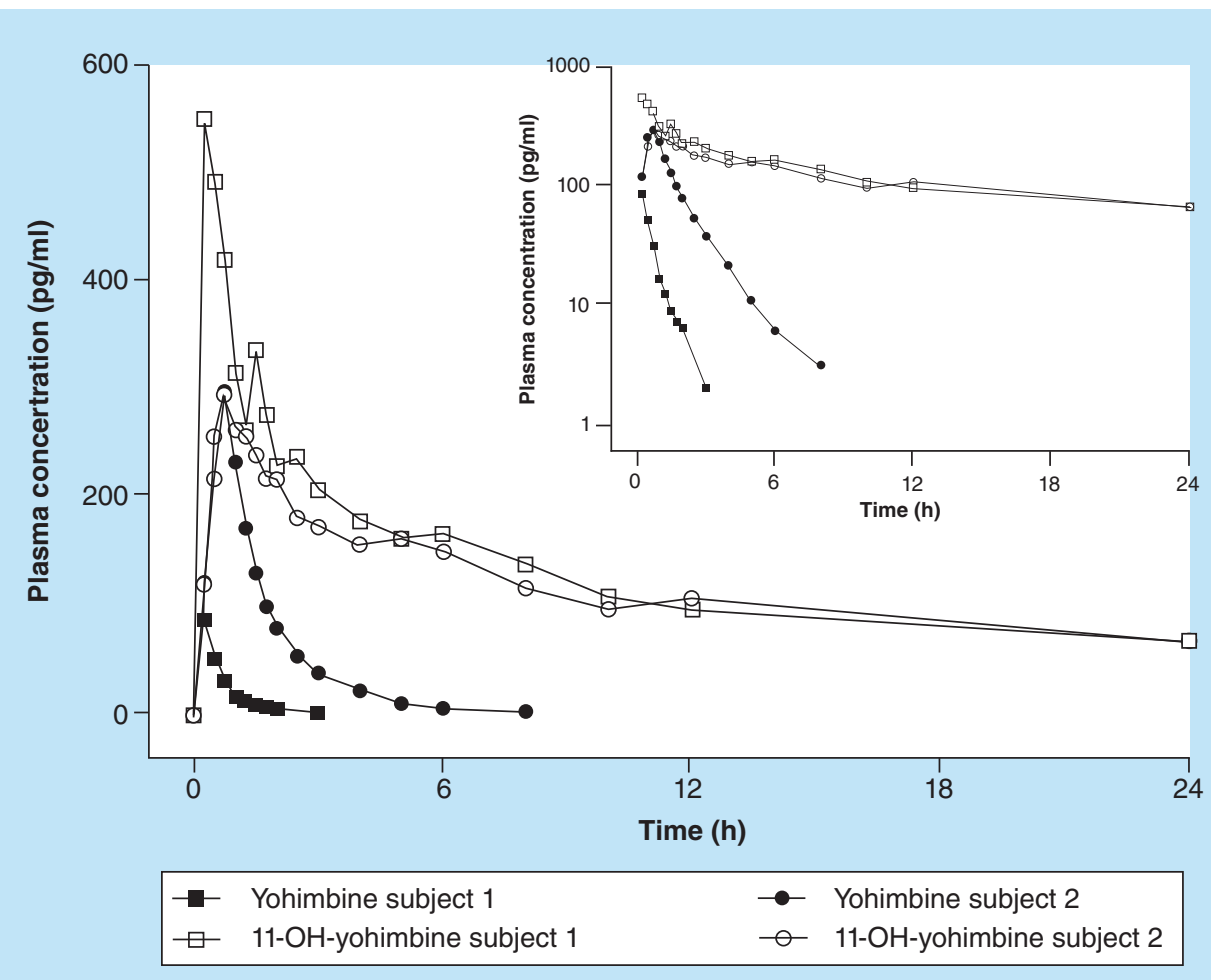

Figure 3. Plasma concentration-time curves of yohimbine and 11-OH-yohimbine after administration of $50 \mu \mathrm{g}$ oral yohimbine to two healthy participants, one with normal CYP2D6 enzyme activity (subject 1) and one with impaired CYP2D6 enzyme activity (subject 2).

\begin{tabular}{|c|c|c|c|c|c|}
\hline Analyte & $c_{\max }(p g / m l)$ & $t_{\max }(p g / m l)$ & $t_{1 / 2}(h)$ & $\mathrm{Cl} / \mathrm{F}(\mathrm{I})$ & V/F (I) \\
\hline \multicolumn{6}{|c|}{ Subject 1: Normal CYP2D6 activity } \\
\hline Yohimbine & 87.3 & 0.25 & 0.67 & 13921 & 806 \\
\hline 11-OH-yohimbine & 551 & 0.25 & 15.1 & & \\
\hline \multicolumn{6}{|c|}{ Subject 2: Impaired CYP2D6 activity } \\
\hline 11-OH-yohimbine & 296 & 0.75 & 17.5 & & \\
\hline
\end{tabular}

faster metabolization of yohimbine in subject 1. This higher enzyme activity is further visual in the shorter pharmacokinetic elimination half-life of yohimbine in subject $1(0.67 \mathrm{~h})$ also indicating major metabolism of yohimbine via CYP2D6.

Hence, the assay is suitable for the investigation of the variable plasma concentrations depending on the CYP2D6 enzyme activity after administration of oral microdoses of yohimbine, which enables evaluation of yohimbine for profiling of CYP2D6 activity. This further allows the application in microdose substance cocktails [13] aiming at the simultaneous determination of multiple CYP enzyme activities, for example, midazolam for CYP3A4 [14,15].

\section{Conclusion}

We developed and validated an ultrasensitive assay for the determination of yohimbine and its major metabolite 11-OH-yohimbine in human plasma using UPLC-MS/MS. Due to its high sensitivity, the assay allowed determination of 11-OH-yohimbine plasma concentrations from a healthy subject after oral administration of yohimbine microdoses for $24 \mathrm{~h}$. Previously described methods only deal with plasma concentrations after administration of pharmacologically active doses (at least $5 \mathrm{mg}$ ), sample volumes of $0.5-2 \mathrm{ml}$ and LLOQs of $0.05-0.2 \mathrm{ng} / \mathrm{ml}$ for 
yohimbine [10] and $1.0 \mathrm{ng} / \mathrm{ml}$ for 11-OH-yohimbine [6]. The straight forward sample preparation combined with small sample volume $(100 \mu \mathrm{l})$ and short run time of the assay is suitable and effective for measurement of high sample numbers in drug-drug interaction trials. It is, to our knowledge, by far the most sensitive assay for quantification of yohimbine and its major metabolite 11-OH-yohimbine enabling determination of pharmacokinetics after oral microdosing. Moreover, judging from the plasma concentration versus time curves, this assay provides a basis for further investigation of possible CYP2D6 enzyme activity prediction using oral microdoses of yohimbine as well as in microdose cocktails for simultaneous profiling of multiple CYP enzyme activities.

\section{Future perspective}

Microdosed yohimbine may be used in drug-drug interaction trials to evaluate CYP2D6 enzyme activity as well as for phenotyping. The possibility to use yohimbine in microdose 'cocktails' for simultaneous characterization of multiple cytochrome P450 enzyme activities in drug-drug interaction trials can further simplify safe pharmacological investigations.

\section{Executive summary}

\section{Background}

- Investigation of the pharmacokinetics of oral microdosed yohimbine for drug-drug interaction trials using UPLC-MS/MS quantification in the picogram range.

Materials \& methods

- Low amount $(100 \mu \mathrm{l})$ of plasma was used and efficient extraction achieved by fast liquid-liquid extraction.

- Ultrasensitive quantification of yohimbine and its major metabolite (LLOQ: $5 \mathrm{pg} / \mathrm{ml}$ ).

- Pharmacokinetics of yohimbine and its metabolite were evaluated in two subjects with different CYP2D6 enzyme activity.

\section{Results \& discussion}

- The UPLC-MS/MS assay was validated in accordance with US FDA's and EMA's pertinent guidelines showing good accuracy and precision.

- The assay was successfully applied to determine yohimbine and 11-OH-yohimbine pharmacokinetics after oral administration of $50 \mu \mathrm{g}$ yohimbine in two healthy subjects.

Conclusion

- The developed assay allows sensitive determination of yohimbine and its metabolite 11-OH-yohimbine in the picogram range after oral microdoses.

- Yohimbine microdosing combined with ultrasensitive quantification is potentially suitable for profiling of CYP2D6 enzyme activity.

Author contributions

G Mikus and J Burhenne conceived the study and supported data analysis. M Vay developed the assay, performed the measurements, conducted the clinical trial, evaluated the data and wrote the manuscript. M Sauter supported assay development, writing of the manuscript and data analysis.

\section{Acknowledgments}

The authors would like to thank M Longo, A Deschlmayr and K Steimel for their valuable assistance during assay development, $\mathrm{P}$ Rose for medical advice, as well as J Fahy and F Sautel from Pierre Fabre for their kind donation of the 11-OH-yohimbine.

Financial \& competing interests disclosure

The authors have no relevant affiliations or financial involvement with any organization or entity with a financial interest in or financial conflict with the subject matter or materials discussed in the manuscript. This includes employment, consultancies, honoraria, stock ownership or options, expert testimony, grants or patents received or pending, or royalties.

No writing assistance was utilized in the production of this manuscript.

Ethical conduct of research

The trial was conducted in the clinical research unit at the Department of clinical pharmacology and pharmacoepidemiology (KliPS, certified according to ISO 9001:2015) and was approved by the local ethics committee (AFmo-496/2017) and legal authority. The trial was conducted in accordance with good clinical practice guidelines, Declaration of Helsiniki and German legal requirements and written consent of the healthy volunteer was obtained. 


\section{References}

Papers of special note have been highlighted as: $\bullet$ of interest

1. Tam SW, Worcel M, Wyllie M. Yohimbine: a clinical review. Pharmacol. Ther. 91(3), 215-243 (2001).

- Summarizes the information on the pharmacokinetics of yohimbine.

2. Berlan M, Montastruc JL, Lafontan M. Pharmacological prospects for $\alpha 2$-adrenoceptor antagonist therapy. Trends Pharmacol. Sci. 13(7), 277-282 (1992).

3. Saenz de Tejada I, Kim NN, Goldstein I, Traish AM. Regulation of pre-synaptic alpha adrenergic activity in the corpus cavernosum. Int. J. Impot. Res. 12(Suppl. 1), S20-S25 (2000).

4. Cohen PA, Wang YH, Maller G, DeSouza R, Khan IA. Pharmaceutical quantities of yohimbine found in dietary supplements in the USA. Drug Test. Anal. 8(3-4), S357-S369 (2015).

5. Le Corre P, Dollo G, Chevanne F, Le Verge R. Biopharmaceutics and metabolism of yohimbine in humans. Eur. J. Pharm. Sci. 9(1), 79-84 (1999).

- Metabolism of yohimbine.

6. Le Verge R, Le Corre P, Chevanne F, Doe De Maindreville M, Royer D, Levy J. Determination of yohimbine and its two hydroxylated metabolites in humans by high-performance liquid chromatography and mass spectral analysis. J. Chromatogr. 574(2), 283-292 (1992).

- Description of the major metabolite of yohimbine.

7. Berlan M, Le Verge R, Galitzky J, Le Corre P. Alpha 2-adrenoceptor antagonist potencies of two hydroxylated metabolites of yohimbine. Br. J. Pharmacol. 108(4), 927-932 (1993).

8. Le Corre P, Parmer RJ, Kailasam MT et al. Human sympathetic activation by alpha2-adrenergic blockade with yohimbine: bimodal, epistatic influence of cytochrome P450-mediated drug metabolism. Clin. Pharmacol. Ther. 76(2), 139-153 (2004).

- Evaluation of the involved CYP-enzymes in yohimbine metabolism.

9. Zhou SF. Polymorphism of human cytochrome P450 2D6 and its clinical significance: part I. Clin. Pharmacokinet. 48(11), 689-723 (2009).

- Overview of the polymorphism of CYP2D6 and implications for drug-drug interactions.

10. Owen JA, Nakatsu SL, Fenemore J, Condra M, Surridge DH, Morales A. The pharmacokinetics of yohimbine in man. Eur. J. Clin. Pharmacol. 32(6), 577-582 (1987).

11. FDA. Guidance for industry: bioanalytical method validation (2018). www.fda.gov/files/drugs/published/Bioanalytical-Method-Validation-Guidance-for-Industry.pdf

12. EMA. Bioanalytical method validation (2011). www.ema.europa.eu/en/documents/scientific-guideline/guideline-bioanalytical-method-validation_en.pdf

- $\quad$ Probe drug cocktail for drug-drug interaction trials.

13. Mikus G. Probes and cocktails for drug-drug interaction evaluation: the future is microdosing? Clin. Pharmacol. Ther. 105(6), 1335-1337 (2019).

- CYP3A4 phenotyping using microdoses.

14. Hohmann N, Kocheise F, Carls A, Burhenne J, Haefeli WE, Mikus G. Midazolam microdose to determine systemic and pre-systemic metabolic CYP3A activity in humans. Br. J. Clin. Pharmacol. 79(2), 278-285 (2015).

15. Burhenne J, Halama B, Maurer M et al. Quantification of femtomolar concentrations of the CYP3A substrate midazolam and its main metabolite $1^{\prime}$-hydroxymidazolam in human plasma using ultra performance liquid chromatography coupled to tandem mass spectrometry. Anal. Bioanal. Chem. 402(7), 2439-2450 (2012). 
